Available online on 15.11.2016 at http://jddtonline.info
(c) 2016, publisher and licensee JDDT, This is an Open Access article which permits unrestricted
noncommercial use, provided the original work is properly cited

Review Article

\title{
FUNDAMENTAL ASPECTS IN THE DEVELOPMENT OF MICROEMULSION SYSTEMS CONTAINING AMPHOTERICIN B FOR OPHTHALMIC USE
}

\author{
Chirlekar, Rohan*, Chaudhari, Dinesh R. \\ Bombay College of Pharmacy, Kalina, Santacruz (E), Mumbai, Maharashtra 400098, India
}

\begin{abstract}
This is an overview of the development of microemulsion systems containing amphotericin B for ophthalmic use. The amphotericin $\mathrm{B}(\mathrm{AmB})$ is used to treat a fungal infection of eye and bioavailability of the AmB is the challenge for the research scientist. The poor bioavailability of $\mathrm{AmB}$ is due mainly to the corneal barrier, which eventually leads to a precorneal loss and subsequent decrease in the absorption of this drug into the intraocular tissues. Also, AmB has limited permeation across the intact corneal epithelium, which along with the toxicity associated with this molecule, epitomizes a major drawback to its clinical use. Therefore, new effective and safe drug vehicles for ocular delivery of AmB are urgently required. Recently, microemulsions (MEs) have been used in ophthalmic drug delivery system owing to their thermodynamic stability, transparent appearance, and favorable viscosity. Information and proper understanding of the physical chemistry and process of formation of AmB-containing MEs would provide reliable information on the best conditions for the use of these systems as eye drops. The aim of this research was thus to review the main studies on the use of MEs as delivery systems for AmB in topical eye treatment.
\end{abstract}

Keywords: Amphotericin B, Eye, Microemulsions, Ophthalmic Solutions.

*Corresponding author

Rohan Chirlekar, Bombay College of Pharmacy, Kalina, Santacruz (E), Mumbai, Maharashtra 400098, India,

Email: chirlekar.rohan@gmail.com

Article Info

Received 24 Oct 2016; Review Completed 04 Nov 2016; Accepted 04 Nov 2016, Available online 15 Nov 2016

URI: http://jddtonline.info/index.php/jddt/article/view/1345

Cite this article as: Chirlekar R, Chaudhari DR, Fundamental aspects in the development of microemulsion systems containing amphotericin B for ophthalmic use, Journal of Drug Delivery \& Therapeutics. 2016; 6(6):54-59 DOI: http://dx.doi.org/10.22270/jddt.v6i6.1345

\section{INTRODUCTION}

Ocular diseases are mainly treated by topically applied drug administered by eye drops. These conventional dosage forms correspond to $90 \%$ of the available ophthalmic formulations, however, the rapid pre-corneal loss associated with nasolacrimal drainage and the presence of tears make this fact one of the major problems associated with the absorption of drugs in the eye $^{1,2}$. Nanotechnology is emerging as a promising alternative for developing a large range of drug delivery systems for the treatment of diseases via the ocular route $^{3}$. These systems, called colloidal mostly can substantially aid in current therapy which employs periocular route as the route of administration ${ }^{3,4}$.
The treatment of conditions such as keratitis caused by fungal infectious agents leads to serious suppurative infections, eventually leading to ulcerations in the involved tissues and have a generally grim prognosis, and the treatment depends on the rapid and efficient diagnosis. However, in addition to the challenges posed by the anatomical nature of the eye, the treatment of these pathologies still generates uncertainty about the clinical protocols to be established ${ }^{5}$.

Amphotericin B (AmB) is a polyene antifungal agent, broad - spectrum of action, which is widely used in the treatment of systemic and ocular fungal infections ${ }^{6}$, 7 . The drug consists of an orange-yellow powder which 
has two important physicochemical properties: an amphiphilic behavior due to the nonpolar and polar groups of the lactone ring and an amphoteric behavior due to the presence of ionizable carboxyl and amino groups. Due to these characteristics, AmB molecule is poorly soluble in aqueous solvents and many organic solvents, and its solubility in water at physiological $\mathrm{pH}$ (pH 6-7) of less than $1 \mathrm{mg} / \mathrm{ml}^{8}$.

The current formulation of AmB (Fungizone ${ }^{\circledR}$ ) contains sodium deoxycholate (surfactant needed to promote micellization of AmB) causes during instillation, severe pain (irritability) which lead to non-adherence to the treatment by the patient and consequently, worsening of symptoms ${ }^{9}$. This toxicity associated with the topical ocular administration, may develop through blepharitis, keratitis, and iritis that according to the literature, relates to this surfactant and can be significantly reduced when the lipid formulations are used as vehicles for the incorporation of the drug without the use of this substance ${ }^{10,11}$.

The aim of this review article is, therefore, to address the main aspects of the development and preparation of microemulsions (MEs) as delivery systems for AmB through via topical ocular administration ${ }^{12-14}$.

\section{Development and preparation of microemulsions for use in the eye}

Although discovered since 1928, the potential of EMs as drug delivery systems for the ophthalmic route has only been explored in the past twenty years ${ }^{15}$. Moreover, as mentioned earlier, the eye is not an easily accessible organ mainly due to the constant presence of nasolacrimal drainage and corneal barrier ${ }^{16}$. In this context, the MEs are presented as an interesting therapeutic alternative for the ophthalmic route. In fact, MEs have a great capacity for delayed action based on the adsorption of nano-droplets, therefore, will not be deleted by the nasolacrimal drainage. In such systems, the cornea will act as a reservoir molecules increasing its bioavailability ${ }^{15}$.

The MEs also correspond to a promising alternative to reduce the adverse effects caused by $\mathrm{AmB}^{17,}{ }^{18}$. These systems are composed of two immiscible liquids, transparent (translucent isotropic) and thermodynamically stable because they are stabilized by an interfacial film of surfactant localized at the oil / water interface ${ }^{19}$. ME yet have additional advantages such as ease of production and sterilization, increased the ability to incorporate both hydrophilic drugs such as lipophilic, small droplets of internal phase viscosity suitable for the release of drugs in the eye, high surface area, ultra-low interfacial tension, high stability and $\operatorname{low}^{15,20-22}$.

To develop and prepare these systems, many factors must be considered according to the desired type of ME, which in turn is determined during the development of pseudo-ternary phase diagrams (DFPT).The factors that lead to thermodynamic stability, which influence the release of the formulation of pharmaceuticals ${ }^{23}$, the requirements concerning the necessary components and potential uses and limitations of a microemulsion system by the ocular route must, therefore, be taken into consideration $^{24-26}$.

As regards the necessary ingredients, ${ }^{15}$ described the use of various substances considered biocompatible for use by this route of administration, emphasizing its main characteristics and purposes, as its implementation and use, specifically the oil and aqueous phases ${ }^{15}$. In this article, there is a description of the main surfactants that can be part of the formulations, as well as its restrictions. In fact, on an $\mathrm{ME}$, the surfactants serve not only to stabilize the system thermodynamically, but also to facilitate the incorporation of the proposed drug. From a physiological point of view, the surfactant may contribute to the drug absorption in the eye by ensuring cornea, with that, increased homogenization of the system with the cell layers and better contact with the epithelium, causing greater absorption thereof into tissues later. On the other hand, should be balanced for high concentrations of surfactants are not used, thus avoiding the occurrence of local toxicity ${ }^{15}$.

From a technological point of view, the MEs can be formulated in three types: oil in water $(\mathrm{O} / \mathrm{W})$, water in oil $(\mathrm{W} / \mathrm{O})$ or bicontínuos systems ${ }^{27}$. To form these systems, in particular, two aspects should be taken into account in the choice of components: the oil and the quantity in the formulation and the hydrophiliclipophilic balance (HLB) of surfactants involved ${ }^{21,28}$.

For the development of a simple microemulsion system, the preparation and study of DFPT are sorely needed. Usually, a DFPT is constructed to determine the composition of the oil phase, water, and surfactants that will be part of ME. Water, oil and a ratio of two surfactants are represented at each corner of the diagram, these being corresponding to $100 \%$ of the particular components. The proportions of each component are therefore described using these diagrams, and they must be combined to form clear and transparent systems, consisting of an important feature for the use of such formulations through the ocular use $^{24}$.

The ME of the formation process requires, first, the determination of the ME region of DFPT, in which can be observed by viewing various mixtures of surfactants and the oil and water phases used ${ }^{15}$.

The first process emphasizes the ME's ability to form spontaneously. They can be prepared in a single step by simple mixing of the components in their ideal proportions. The order of addition of components is not regarded as a crucial factor in the formation process, but can influence the time necessary to achieve equilibrium $^{15}$.

In the process with power supply, the ME is not formed spontaneously. In this process, a decrease in the amount of surfactants, and sometimes necessary, results in the necessity of using high-pressure homogenizers, in order to obtain droplets of a desirable size, which constitute the internal phase and to have a higher speed the process of formation ${ }^{15}$. 
The development of new systems for ocular use, the formulator must consider multiple perspectives. In addition to the physiological barriers imposed by the eye and shortcomings of conventional dosage forms to promote adequate absorption, many drugs will not have favorable characteristics for use by this route and due to their clinical importance in the treatment of various diseases; they continue to be administered by adverse patient conditions. Thus, after the choice of likely microemulsion systems from DFPT, it is necessary to characterize these systems with and without the drug in order to obtain a formulation encompassing all ocular administration route requirements and favoring clinical and necessary technological ${ }^{29}$. One final aspect to be considered in the development of EMs for the ocular route is its safety regarding eye irritation and possible pre-corneal retention. Alany et al ${ }^{30}$, studied this point and the assumption that the phase transition of an ME, induced by the presence of tears, turning into liquid crystals could prolong the pre-corneal retention of molecules. They came to the conclusion that this phenomenon can effectively occur, but that the MEs have nevertheless a great protective effect compared with aqueous solutions and can, therefore, be used as carriers in the principles of active hydrophilic high irritant power ${ }^{30}$.

\section{INCORPORATION OF AMB}

Many problems related to the administration of $\mathrm{AmB}$ to give local effect in the eye. Intravenous administration is often related to the control of fungal infections in posterior segment (endophthalmitis) ${ }^{31}$, but severe renal toxicity associated with systemic effect and small intraocular penetration limits its use in higher concentrations ${ }^{32-34}$. The conjunctival administration of AmB causes severe necrosis at the site of application, thus its use is not recommended ${ }^{10}$. On the other hand, the intravitreal administration of $\mathrm{AmB}$ is associated with the possible toxicity of the retina and its possible displacement if its application is not held exactly in the center of the vitreous humor ${ }^{32}$. However, intracameral administration of $\mathrm{AmB}$ has been proven effective in the treatment of lesions with subsequent appearance of minimal toxicity reactions however the efficiency of this mode of administration is hampered by a lack of further trials. All of these forms, commonly performed in the clinic, bring inconveniences to the patients who need them and not always results are shown satisfactory ${ }^{35-38}$.

The use of carrier lipid to incorporate AmB has been described in recent years by several groups, but none of them assigned the job of ME to ocular ${ }^{6,17,18}$. The physicochemical characteristics of EMs, with the aim of carrying AmB, can be adapted to the point of promoting its use through this route and consist in future in a new system able to be administered by applying eyedrops ${ }^{29}$.

Despite the intrinsic characteristics of AmB, incorporation of this drug can be difficult. Despite its low solubility in water and in oil (amphoteric nature) under physiological $\mathrm{pH}$ conditions, the use of alkaline solutions has been shown to promote solubilization of the drug and can easily be used ${ }^{17-19,29}$.
The AmB content of microemulsion systems can be determined by using analytical methods such as highperformance liquid chromatography and by reverse phase spectrophotometry. The results determined by such techniques show that the AmB can be easily detected when incorporated into MEs, being located at the oil-water interface, or even within the droplet oil which forms the internal phase, thus presenting an encapsulation rate (incorporation) around $100 \%{ }^{18,29,39}$.

\section{DETERMINING THE PHYSICOCHEMICAL CHARACTERISTICS}

\section{Ideal macroscopically}

Macroscopic characteristics allow to emphasize that these systems have the requirements of a true ME and the presence of $\mathrm{AmB}$ is or is not able to alter the intrinsic features necessary for an ophthalmic system without the drug presented by the system. The clarity, homogeneity, absence of particulate matter, creaming or phase separation, in addition to transparency and thermodynamic stability correspond to essential factors of the MEs that should remain unchanged even after the addition of the drug ${ }^{40}$.

The classification proposed by Winsor in 1948 to disperse systems, requires single phase, the Winsor IV type is preferable as drug delivery systems. Transparency, clarity, and homogeneity of the phases are features present in EMs and extremely important for the administration through the ocular ${ }^{41}$.

\section{pH range}

The AmB has maximum therapeutic activity in the $\mathrm{pH}$ range between 6.0 and $7.5^{42}$. In addition, this track is very important to promote the necessary comfort to the patient when the formulation is administered in the eye. If the $\mathrm{pH}$ of the composition is outside this range, a possible irritation may arise, with possible damages to the tissues, inducing a greater tearing ${ }^{20,}{ }^{43}$, greater elimination of $\mathrm{AmB}$ compromising its bioavailability and its efficacy in affected tissues ${ }^{44}$. The use of tampons as the aqueous phase consists of a fact very important to ensure that there will be variations in the required $\mathrm{pH}$ range, keeping, thus, the comfort to the patient upon administration and maximum activity of AmB in MEs proposals $^{15}$.

\section{Refractive index and transmittance percentage}

Transparency is a very important factor to be noted, as should the patient avoid the occurrence of blurred vision and discomfort after administration of the microemulsion system in the eye. This can be easily observed in EMs, due to the proximity of their values to the refractive index presented by the tears and the cornea, thus consisting of one of its main advantages for use by ocular route 21,45 . The transmittance percentage above $99 \%$ corroborates this parameter together and prove that the system really has obtained transparency ${ }^{46}$.

Recently it was demonstrated that the presence of AmB incorporated in an ME is able to partially reduce this transparency. However, such change does not influence the initial system stability and homogeneous and 
transparent characteristic described in Winsor IV classification is thus maintained ${ }^{29}$.

\section{Conductivity}

MEs type $\mathrm{O} / \mathrm{A}$ has been proposed as aqueous vehicles for the release of numerous drug molecules. Conductivity is an important parameter in characterizing the MEs, because it is able to determine the type of ME formed from the combination of its components. This test claims to have an ME aqueous or oily external phase based on the same capacity to conduct electrical current ${ }^{47}$.

The use of BD aqueous outer phase is more appropriate for administration by the ocular route, because during its application, this system is able to mix and distribute tears, maintaining the uniformity of the droplets with the same and ensuring the better release of $\mathrm{AmB}$ in the cornea $^{48}$.

\section{Droplet size and polydispersity index}

The determination of droplet size and its distribution corresponds to one of the most important parameters to evaluate the stability of microemulsion systems and the influence of the incorporation of poorly soluble drugs which could cause instability of the system ${ }^{49}$. The small size of the order of 50 nanometers, characterized ME and it is due to the interaction between the molecules of the cosurfactant to the surfactant film on the internal phase, thus decreasing the radius of curvature of the microdroplets, providing training of transparent systems $^{24,29}$.

Some studies reported in the literature show that the incorporation of AmB the droplet size increases initially originated with the ME without the drug. This is probably the intrinsic physicochemical properties of the molecule (anfotericidade and amphiphilicity) who take the drug to be located within the droplet or even in their interfacial region. However, this fact cannot change the system stability and isotropic properties are therefore retained $^{19,29}$.

\section{Surface tension}

The vast majority of authors who develop microemulsion systems states that the application of a negative free energy is required for the spontaneous formation is optimized. This achievement is accompanied by dramatic reductions in surface tension present between the aqueous and oil phase. For this to be provided, high surfactant concentrations should be used by increasing thereby the risk of toxicity ${ }^{48,50}$.

In some cases, it is necessary to decrease the amount of surfactants and increase the proportion of the aqueous phase to obtain a given microemulsion system. Thus, probably surface tension show variations and this will require the formulator the use of other methodologies system training, as well as the use of other devices for the incorporation of drugs which require higher concentrations of these substances, exert their $\operatorname{activities}^{15}$.
The surface tension should therefore not be influenced by the addition of the drug, it being essential for the maintenance of the thermodynamic stability of the ME before and after incorporation and must remain the best ratio between components ${ }^{29}$.

\section{Zeta potential}

The human corneal surface is negatively charged, so the use of internal phase microemulsion systems which present positive charge can directly influence the absorption of AmB by topically. Studies have shown that positively charged PMs are suitable for use by this route. The electrostatic attraction between positively charged PMs with the negative surface of the cornea generates a bioadhesion due to the connection between the opposite charges, causing an increase in drug absorption. Therefore, the development of PMs with zeta potential suitable for topical administration would act by increasing the $\mathrm{AmB}$ time of contact with the corneal surface favoring their absorption and thereby increasing the biodisponililidade the posterior tissues of the eye $\mathrm{e}^{51,52}$.

\section{Isotonic}

The use of isotonizing agents should be contained in the aqueous phase as well as the use of buffering agents $^{53}$. The need to formulate isotonic systems for the eyes is also linked to comfort the pharmaceutical form will give the patient when it is administered. However, one should be very careful, because the use of salinizantes agents may decrease the temperature of phase inversion of surfactants causing a change in the external phase system promoting a decrease of stability in concomitant use with ionic surfactants ${ }^{15}$.

\section{Viscosity and rheology}

Ocular therapy with AmB - containing systems could be significantly improved if the pre-corneal contact time is increased. The increase in contact time can provide increased absorption of drugs through the cornea and to reduce the systemic adverse effects displayed by conventional dosage forms ${ }^{54}$. The expected viscosity values must be present above those that are observed for conventional ophthalmic solutions (approximately 20 $\mathrm{MPa}$ ) in order to provide a higher AMB retention time of the corneal surface and allow a greater penetration of the drug into the eye $\mathrm{e}^{21}$.

The determination of viscosity and rheological behavior in EMs is required to physically characterize and control their stability ${ }^{17}$. These parameters are important when the molecule is administered by ocular route. 0 The presence of $\mathrm{AmB}$ incorporated into the system should not cause changes in rheological behavior as occasional changes would provide problems with respect to the ocular administration and stability of the system itself. There is also the system, depending on the purpose of the formulator can present several rheological behaviors, the most important being the Newtonian. This behavior ensures that the formulations will not change in viscosity with the tearing and the action of the eye blink. 
The viscosity values should allow further that the systems can be sterilized by filtration, a factor essential in the development of pharmaceutical forms for ocular administration, as it ensures the absence of any microbiological contamination ${ }^{21}$.

\section{CONCLUSION}

The development of new systems for the carrier AmB to improve its bioavailability and reduce its toxicity as well as the drawbacks presented with the administration of conventional forms of a pharmaceutical formulation (Fungizone $^{\circledR}$ ), if necessary. In the near future, emphasis should be given for the treatment of ocular disorders using a non-invasive molecules delivery system and capable of reaching all segments of the eye. In this context, MEs appear as a promising system since they possess physicochemical properties that allow its use over the ocular route. MEs also consist of suitable systems incorporating other poorly soluble drugs, becoming a new alternative for the treatment of superficial fungal infections and other internal disorders that affect the eye. The use of MEs containing AmB (AmB ME) should be able to maintain efficacy, improve bioavailability and reduce the toxicity observed for the $\mathrm{AmB}$ when used via the intravenous and ocular routes in control of systemic fungal infections and ocular.

\section{CONFLICT OF INTEREST}

The authors report no conflict of interest.

\section{REFERENCES}

1. Carlfors J, Edsman K, Petersson R, Jörnving K, Rheological evaluation of Gelrite ${ }^{\circledR}$ in situ gels for ophthalmic use, European journal of pharmaceutical sciences, 1998, 6(2), 113-9.

2. Parekh HB, Jivani R, Jivani N, Patel L, Makwana A, Sameja $\mathrm{K}$, Novel insitu polymeric drug delivery system: a review, Journal of Drug Delivery and Therapeutics, 2012; 2(5):136145.

3. Gaudana R, Jwala J, Boddu SH, Mitra AK, Recent perspectives in ocular drug delivery, Pharmaceutical research, 2009, 26(5), 1197-216.

4. Bonacucina G, Cespi M, Misici-Falzi M, Palmieri GF, Colloidal soft matter as drug delivery system, Journal of pharmaceutical sciences, 2009, 98(1), 1-42.

5. Chaumeil C, Bourcier T, Rostane H, et al., Diagnosis and treatment of keratomycosis and fungal endophthalmitis, JOURNAL DE MYCOLOGIE MEDICALE, 2007, 17(2), 89108.

6. Esposito E, Bortolotti F, Menegatti E, Cortesi R, Amphiphilic association systems for Amphotericin B delivery, International journal of pharmaceutics, 2003, 260(2), 249-60.

7. Wood T, Tuberville A, Monnett R, Keratomycosis and amphotericin B, Transactions of the American Ophthalmological Society, 1985, 83, 397.

8. Torrado J, Espada R, Ballesteros M, Torrado-Santiago S, Amphotericin B formulations and drug targeting, Journal of pharmaceutical sciences, 2008, 97(7), 2405-25.

9. Morand K, Bartoletti A, Bochot A, Barratt G, Brandely M, Chast F, Liposomal amphotericin B eye drops to treat fungal keratitis: physico-chemical and formulation stability, International journal of pharmaceutics, 2007, 344(1), 150-3.

10. Kaji Y, Yamamoto E, Hiraoka T, Oshika T, Toxicities and pharmacokinetics of subconjunctival injection of liposomal amphotericin B, Graefe's Archive for Clinical and Experimental Ophthalmology, 2009, 247(4), 549-53.
11. Mohan K, Pravin S, Atul B, Ophthalmic microemulsion: a comprehensive review, Int J Pharm Bio Sci, 2012, 3(3), 113.

12. Saini JK, Nautiyal U, Kumar M, Singh D, Anwar F, Microemulsions: A potential novel drug delivery system, International Journal of Pharmaceutical and Medicinal Research, 2014, 2(1), 15-20.

13. Hashem FM, Shaker DS, Ghorab MK, Nasr M, Ismail A, Formulation, characterization, and clinical evaluation of microemulsion containing clotrimazole for topical delivery, AAPS PharmSciTech, 2011, 12(3), 879-86.

14. Hegde RR, Verma A, Ghosh A, Microemulsion: new insights into the ocular drug delivery, ISRN pharmaceutics, 2013, 2013.

15. Vandamme TF, Microemulsions as ocular drug delivery systems: recent developments and future challenges, Progress in retinal and eye research, 2002, 21(1), 15-34.

16. Bottos KM, Oliveira AG, Bersanetti PA, et al., Corneal absorption of a new riboflavin-nanostructured system for transepithelial collagen cross-linking, PLoS one, 2013, 8(6), e66408.

17. Brime B, Moreno MA, Frutos G, Ballesteros M, Frutos P, Amphotericin B in oil-water lecithin-based microemulsions: Formulation and toxicity evaluation, Journal of pharmaceutical sciences, 2002, 91(4), 1178-85.

18. Moreno MA, Frutos P, Ballesteros MP, Lyophilized lecithin based oil-water microemulsions as a new and low toxic delivery system for amphotericin B, Pharmaceutical research, 2001, 18(3), 344-51.

19. Pestana K, Formariz T, Franzini C, et al., Oil-in-water lecithin-based microemulsions as a potential delivery system for amphotericin B, Colloids and surfaces B: Biointerfaces, 2008, 66(2), 253-9.

20. Gupta S, Moulik S, Biocompatible microemulsions and their prospective uses in drug delivery, Journal of pharmaceutical sciences, 2008, 97(1), 22-45.

21. Fialho SL, Silva-Cunha D, New vehicle based on a microemulsion for topical ocular administration of dexamethasone, Clinical \& experimental ophthalmology, 2004, 32(6), 626-32.

22. Dwivedi C, Sahu R, Tiwari SP, Satapathy T, Roy A, Role of liposome in novel drug delivery system, Journal of Drug Delivery and Therapeutics, 2014, 4(2):116-129.

23. Chaudhari SP, Dave RH, Investigating the Effect Of Molecular Weight of Polyvinylpyrrolidone and Hydroxypropyl Methyl Cellulose as Potential Antiprecipitants on Supersaturated Drug Solutions and Formulations using Weakly Acidic Drug: Indomethacin, International Journal of Pharmaceutical Sciences and Research, 2016, 7(10), 3931-48.

24. Tenjarla S, Microemulsions: an overview and pharmaceutical applications, Critical Reviews $^{\mathrm{TM}}$ in Therapeutic Drug Carrier Systems, 1999, 16(5).

25. Kaur IP, Rana C, Singh H, Development of effective ocular preparations of antifungal agents, Journal of Ocular Pharmacology and Therapeutics, 2008, 24(5), 481-94.

26. Muzaffar F, Singh U, Chauhan L, Review on microemulsion as futuristic drug delivery, Int J Pharm Pharm Sci, 2013, 5(3), 39-53.

27. Silva AE, Barratt G, Chéron M, Egito EST, Development of oil-in-water microemulsions for the oral delivery of amphotericin B, International journal of pharmaceutics, 2013, 454(2), 641-8.

28. Bouchemal K, Briançon S, Perrier E, Fessi H, Nanoemulsion formulation using spontaneous emulsification: solvent, oil and surfactant optimisation, International journal of pharmaceutics, 2004, 280(1), 241-51.

29. Damasceno BP, Dominici VA, Urbano IA, et al., Amphotericin B microemulsion reduces toxicity and maintains the efficacy as an antifungal product, Journal of biomedical nanotechnology, 2012, 8(2), 290-300.

30. Alany R, Rades T, Nicoll J, Tucker I, Davies N, W/O microemulsions for ocular delivery: Evaluation of ocular 
irritation and precorneal retention, Journal of controlled release, 2006, 111(1), 145-52.

31. Kumar JR, Muralidharan S, Parasuraman S, Antifungal agents: New approach for novel delivery systems, 2014.

32. Thomas PA, Current perspectives on ophthalmic mycoses, Clinical microbiology reviews, 2003, 16(4), 730-97.

33. Yamaguchi M, Yasueda S-i, Isowaki A, et al., Formulation of an ophthalmic lipid emulsion containing an antiinflammatory steroidal drug, difluprednate, International journal of pharmaceutics, 2005, 301(1), 121-8.

34. Chaudhari SP, Dave RH, Evaluating the Effects of Different Molecular Weights of Polymers in Stabilizing Supersaturated Drug Solutions and Formulations Using Various Methodologies of the Model Drug: Fenofibrate, Journal of Pharmaceutical Sciences and Pharmacology, 2015, 2(3), 259-76.

35. Kaushik S, Ram J, Brar GS, Jain AK, Chakraborti A, Gupta A, Intracameral amphotericin B: initial experience in severe keratomycosis, Cornea, 2001, 20(7), 715-9.

36. Kuriakose T, Kothari M, Paul P, Jacob P, Thomas R, Intracameral amphotericin $\mathrm{B}$ injection in the management of deep keratomycosis, Cornea, 2002, 21(7), 653-6.

37. Yilmaz S, Ture M, Maden A, Efficacy of intracameral amphotericin B injection in the management of refractory keratomycosis and endophthalmitis, Cornea, 2007, 26(4), 398-402.

38. Yoon K-C, Jeong I-Y, Im S-K, Chae H-J, Yang S-Y, Therapeutic effect of intracameral amphotericin B injection in the treatment of fungal keratitis, Cornea, 2007, 26(7), 814-8.

39. Tejpal Y, Jat R, Microspheres as an ocular drug delivery system-A review, J Drug Deliv Therap, 2013, 3, 114-23.

40. Narang AS, Delmarre D, Gao D, Stable drug encapsulation in micelles and microemulsions, International journal of pharmaceutics, 2007, 345(1), 9-25.

41. Winsor P, Hydrotropy, solubilisation and related emulsification processes, Transactions of the Faraday Society, 1948, 44, 376-98.

42. Kaur IP, Kakkar S, Topical delivery of antifungal agents, Expert opinion on drug delivery, 2010, 7(11), 1303-27.

43. Honda M, Asai T, Oku N, Araki Y, Tanaka M, Ebihara N, Liposomes and nanotechnology in drug development: focus on ocular targets, International journal of nanomedicine, 2013, 8, 495.

44. Ali Y, Lehmussaari K, Industrial perspective in ocular drug delivery, Advanced drug delivery reviews, 2006, 58(11), 1258-68.

45. Amal El Sayeh F, Hassan MA, El-Maraghy DA, Ketorolac tromethamine floating beads for oral application: characterization and in vitro/in vivo evaluation, Saudi Pharmaceutical Journal, 2014, 22(4), 349-59.

46. Ghosh PK, Majithiya RJ, Umrethia ML, Murthy RS, Design and development of microemulsion drug delivery system of acyclovir for improvement of oral bioavailability, AAPS PharmSciTech, 2006, 7(3), E172-E7.

47. Karasulu HY, Microemulsions as novel drug carriers: the formation, stability, applications and toxicity, Expert opinion on drug delivery, 2008, 5(1), 119-35.

48. Lawrence MJ, Rees GD, Microemulsion-based media as novel drug delivery systems, Advanced drug delivery reviews, 2000, 45(1), 89-121.

49. Charman SA, Charman WN, Rogge MC, Wilson TD, Dutko FJ, Pouton CW, Self-emulsifying drug delivery systems: formulation and biopharmaceutic evaluation of an investigational lipophilic compound, Pharmaceutical research, 1992, 9(1), 87-93.

50. Chaudhari SP, Dave RH, To prepare and characterize microcrystalline cellulose granules using water and isopropyl alcohol as granulating agents and determine its end-point by thermal and rheological tools, Drug development and industrial pharmacy, 2015, 41(5), 744-52.

51. Abdulrazik M, Tamilvanan S, Khoury K, Benita S, Ocular delivery of cyclosporin A. II. Effect of submicron emulsion's surface charge on ocular distribution of topical cyclosporin A, STP pharma sciences, 2001, 11(6), 427-32.

52. Tamilvanan S, Khoury K, Gilhar D, Benita S, Ocular delivery of cyclosporin AI Design and characterization of cyclosporin A-loaded positively-charged submicron emulsion, STP pharma sciences, 2001, 11(6), 421-6.

53. Rawas-Qalaji M, Williams C-A, Advances in ocular drug delivery, Current eye research, 2012, 37(5), 345-56.

54. Chan J, El Maghraby GM, Craig JP, Alany RG, Phase transition water-in-oil microemulsions as ocular drug delivery systems: in vitro and in vivo evaluation, International journal of pharmaceutics, 2007, 328(1), 65-71. 